\title{
H1N1 2009 Pandemic - Lessons learnt
}

\author{
Nitin K SHaH \\ Consultant Pediatrician, PD Hinduja National Hospital, Mumbai, India.drnitinshah@hotmail.com
}

$\mathrm{O}$ n 11 June, 2009, WHO declared phase 6 of the 1 st influenza pandemic of the current century caused by pandemic H1N1 2009 strain. On 10 August, 2010, WHO announced that the world was in postpandemic phase, though fresh cases continued to be reported from India till the end of September 2010 $[1,2]$.

Pandemic H1N1 2009 infection, though milder than previous influenza pandemics and with less mortality, affected healthy young people more often than the elderly people; $25-50 \%$ of cases who were hospitalized or died had no co-morbidities [3]. In contrast, in this issue of Indian Pediatrics, Das et al, reported that all children with co-morbidities required hospitalization [4]. In India, 33\% of cases were reported in 5-19 years of age group and $40 \%$ in 20-39 years of age group [2]. Mortality due to pandemic H1N1 2009 has been estimated to be $<0.5 \%(0.0004-1.47 \%)$, but it is difficult to estimate as many cases are mild or sub-clinical giving an incorrect denominator [3]. This is also evident from a seroepidemiology study done from Pune during the 2009 pandemic which showed that the peak seroprevalence was $20 \%$ in hospital staff, $35.6 \%$ in general medical practitioners, $26 \%$ in school staff, $7.7 \%$ in adult general population, $13.2 \%$ in children general population, $28.3 \%$ in $15-19$ years general population and only $3.5 \%$ in those older than 60 years [5]. Media played its role in blowing up the pandemic cases and deaths which created a fear psychosis amongst patients and the treating practitioners alike and highlighted the country's poor preparedness for the pandemic. Transferring a sick patient to a facility with isolation was a nightmare for the practicing pediatricians due to lack of isolation wards. Experts of the IAP (Indian Academy of
Pediatrics), Mumbai branch, prepared local guidelines to manage pandemic influenza following which the Central IAP and the IAP Infectious Disease Chapter adopted IAP guidelines for the same. The Ministry of Health and Family Welfare, Government of India, also put up the National guidelines on the website which clearly defined the categories of suspected pandemic H1N1 2009 cases and guidelines for testing and treatment with oseltamivir [6]. The confusion persisted though with contrary local guidelines for Maharashtra [7]. Dissipation of national guidelines to medical fraternity was a challenge and was partially met by infomercials in media. Efficacy of measures like wearing ordinary face masks and closing of schools was questionable as in Pune, where despite these measures, the virus infected a significant percentage of population. Developing an effective distribution system for oseltamivir was a herculean task and the price in private market remained exorbitant. One positive aspect of the whole pandemic was an increased awareness about personal hygiene, especially hand sanitization, which is practiced by many even today routinely.

World over, monovalent pandemic H1N1 2009 vaccines were available in late 2009 but only to the developed countries. Enough stocks were available only by late August 2010 when they reached India and by then the pandemic was declared as over! IAP COI was prompt in adopting guidelines on the use of pandemic and seasonal 2010 influenza vaccine; and now that the vaccines are available, we are all reluctant to use it thinking that the pandemic is over! Unless we have a national policy on the use of influenza vaccine, the situation will not improve and we will face the same music even during the next pandemic whenever it occurs! There are millions of 
'at risk' persons including children in India who always needed influenza vaccine every year, and IAP has for long recommended influenza vaccine for these 'at risk' individuals, and yet a miniscule of these persons are actually offered the vaccine. The bright side of the story is that indigenous manufacturers from India did come out with a monovalent H1N1 2009 vaccine and hope to follow it up with trivalent influenza vaccine this year. This will go a long way in obviating the dependence on foreign manufacturers and also help bring down the price of influenza vaccines in India in future.

A post-pandemic phase does not mean the end of pandemic H1N1 2009 infection, it only means that the peak of transmission of pandemic H1N1 2009 is over and it is expected that pandemic H1N1 2009 will now co-circulate with other seasonal influenza $A$ and B stains. In the post-pandemic period, cases and outbreaks due to the pandemic H1N1 2009 are expected to continue to occur. In addition, it is most likely that for some period of time, the younger age groups, including pregnant women, will continue to be affected disproportionately by severe disease from pandemic H1N1 2009, including viral pneumonia. Pandemic H1N1 2009 may be down but is certainly not out $[8,9]$. Hence, a continued surveillance for influenza is required by all the countries to monitor the situation.

To conclude; the lessons learnt from influenza pandemic from the the Indian perspective are that we need to be better prepared for the pandemic in future, we need to have a long term policy on influenza surveillance and use of influenza vaccine, we need to be more methodical in our approach to a future outbreak of any infection or medical emergency and we need to have evidence based scientific guidelines to tackle such a medical emergency rather than giving a knee-jerk response in future!

Competing interests: The author received general consultancy fees and speaker's honoraria from Sanofi Pasteur India and GSK India both of which manufacture and market influenza vaccines. Funding: None.

\section{REFERENCES}

1. WHO. Pandemic (H1N1) 2009 - update 112. Available at http://www.who.int/csr/don/2010_08_06/en/index.html. Accessed on December 24, 2010.

2. MOHFW GOI. Situation Update on H1N1. Available at http://mohfw-h1n1.nic.in/documents/PDF/ EpidemiologicalTrendsInIndia.pdf. Accessed on December 24, 2010.

3. Writing Committee of the WHO Consultation on Clinical Aspects of Pandemic (H1N1) 2009 Influenza. Clinical Aspects of Pandemic 2009 Influenza A (H1N1) Virus Infection. N Engl J Med. 2010;362:1708-19.

4. Das RR, Sami A, Lodha R, Jain R, Broor S, Kaushik S, et al. Clinical profile and outcome of swine flu in Indian children. Indian Pediatr. 2010;48:373-8.

5. Tandale BV, Pawar SD, Gurav YK, Chadha MS, Kuratkar MS, Shelke VN et al. Seroepidemiology of pandemic influenza A (H1N1) 2009 virus infection in Pune India. Poster presentation no. P-277 Abstract book of the conference Epidemiology, Disease burden and Health Economics, Options for the control of Influenza infection VII held at Honk Kong, 2010. p. 210.

6. MOHFW GOI. Guidelines on categorization of influenza A H1N1 cases during screening for home isolation, testing, treatment and hospitalization. Available at http://mohfwh1n1.nic.in. Accessed on 4 October, 2009.

7. Shah N. H1N1 guidelines - reply. Indian Pediatr. 2010;47:102-3.

8. Prevention and Control of Influenza with Vaccines Recommendations of the Advisory Committee on Immunization Practices (ACIP), 2010. MMWR. 2010;59:1-64.

9. WHO. Influenza Update -17 December 2010. Available at http://www.who.int/csr/disease/influenza/ 2010_12_17_GIP_surveillance/en/index.html Accessed on 24 December, 2010. 